\title{
SUPORTE ODONTOLÓGICO AO PACIENTE PEDIÁTRICO ONCOLÓGICO NO MUNICÍPIO DE MARINGÁ - PR
}

\author{
Elis Müller \\ Beatriz de Souza da Silva Zamboni Martins* \\ Maria Paula Jacobucci Botelho"*
}

\begin{abstract}
RESUM0: Pacientes oncológicos pediátricos podem se beneficiar de tratamento odontológico antes e durante a terapia do câncer, pois tanto a quimioterapia quanto a radioterapia, podem causar alterações nos tecidos sadios da cavidade oral. 0 objetivo deste projeto foi identificar lesões e alterações bucais em pacientes pediátricos oncológicos cadastrados na Rede Feminina de Combate ao Câncer de Maringá/PR e aplicar métodos de atenção primária e curativa. 0 projeto recebeu aprovação do Comitê de Ética em Pesquisa do UniCesumar (Protocolo ${ }^{0}$ 1.474.425). Foram realizadas 22 visitas domiciliares a esses pacientes, com idades variando entre dois e 14 anos. Nas visitas foram incentivados os hábitos de higiene bucal e dieta saudável, e fornecida uma escova dental para cada uma. Aos pacientes que necessitavam de tratamento curativo, foi oferecido tratamento na Clínica de Odontologia do UniCesumar. Neste estudo foi relatado que os pacientes apresentaram náusea, disfagia, mucosite oral, xerostomia, disgeusia e candidíase oral, durante 0 tratamento oncológico o qual foram submetidas. Com isso foi determinado que a presença do cirurgião-dentista na equipe multidisciplinar oncológica é fundamental.
\end{abstract}

PALAVRAS-CHAVE: Câncer; Lesões; Odontologia pediátrica.

\section{DENTISTRY SUPPORT TO THE PEDIATRIC PATIENT WITH CANCER, IN MARINGÁ, BRAZIL}

ABSTRACT: Children with cancer may be treated for dental problems before and after cancer therapy. In fact, chemotherapy and radiotherapy may affect the healthy tissues of the mouth cavity. Lesions and mouth diseases were identified in children with cancer enrolled on the Feminine Network against Cancer in Maringá PR Brazil, whilst primary and curative care was administered. Current project was approved by the UniCesumar Committee for Ethics in Research. Twenty-two home visits were made to patients aged $2-14$ years old. Mouth hygiene and a healthy diet were encouraged. A tooth brush was given to each. Treatment at the Dental Clinic of UniCesumar was offered to patients requiring treatment. Patients undergoing cancer treatment were committed with nausea, dysphagia, mouth mucositis, xerostomia, dysgeusia and mouth candidiasis during cancer treatment. Results show that the dentist should also take part in the cancer multidisciplinary team.

KEYWORDS: Cancer; Lesions; Pediatric dentistry.

\section{INTRODUÇÃO}

0 câncer infantil ainda apresenta alto índice de incidência e alta mortalidade, sendo a principal causa de morte em crianças com menos de 15 anos de idade, atingindo dez em cada 1.000 .000 de crianças por ano em todo o mundo (INCA, 2011).

Quando um indivíduo recebe 0 diagnóstico de câncer, cuidados adicionais em relação à saúde bucal devem ser estabelecidos, entretanto, nem sempre isso é possível por conta da dificuldade de acesso da família ao tratamento odontológico ou, ainda, pela falta de conhecimento dessa família em relação ao papel do cirurgião-

\footnotetext{
Graduada em Odontologia pelo Centro Universitário de Maringá (UNICESUMAR), Maringá (PR), Brasil.

** Discente de odontologia pelo Centro Universitário de Maringá (UNICESUMAR), Maringá (PR), Brasil.

${ }^{* * *}$ Doutorado em Dentística pela Universidade Norte do Paraná (UNOPAR). Docente titular do Centro Universitário de Maringá (UNICESUMAR). Docente convidada da Pós-graduação Stricto sensu da Universidade Norte do Paraná (UNOPAR), Brasil. E-mail: paulajacobucci@hotmail.com
} 
dentista para a saúde geral.

Ao longo dos anos a eficácia do tratamento do câncer tem proporcionado melhoria na qualidade de vida dos pacientes acometidos por esta doença. No entanto, os danos colaterais para as estruturas de cabeça e pescoço são encontrados frequentemente como uma consequência indesejada. A radioterapia e a quimioterapia podem causar danos diretos aos tecidos moles e duros das estruturas orais e a toxicidade sistêmica pode dar origem a danos indiretos (ALBUQUERQUE, 2007). Estas complicações orais, agudas ou crônicas, podem surgir durante e após 0 tratamento do câncer e, frequentemente, incluem mucosite, disgeusia, xerostomia, doenças infecciosas, disfagia e hipossalivação (HALYARD, 2009; WONG, 2014).

No decorrer do tratamento, as crianças com câncer apresentam sequelas bucais e desconforto (CAMARG0 et al, 2004). Em face disto, considera-se que pacientes oncológicos pediátricos apresentam necessidade de tratamento odontológico antes e durante a terapia do câncer, haja vista que tanto a quimioterapia quanto a radioterapia podem causar alterações nos tecidos sadios da cavidade oral (PAIVA, 2007).

A relevância social deste estudo se destaca do ponto de vista teórico por demonstrar a importância da odontologia como suporte para pacientes oncopediátricos numa perspectiva multiprofissional. Do ponto de vista prático por propor ações de atenção primária odontológica que podem resultar na melhoria da qualidade de vida de pacientes oncopediátricos.

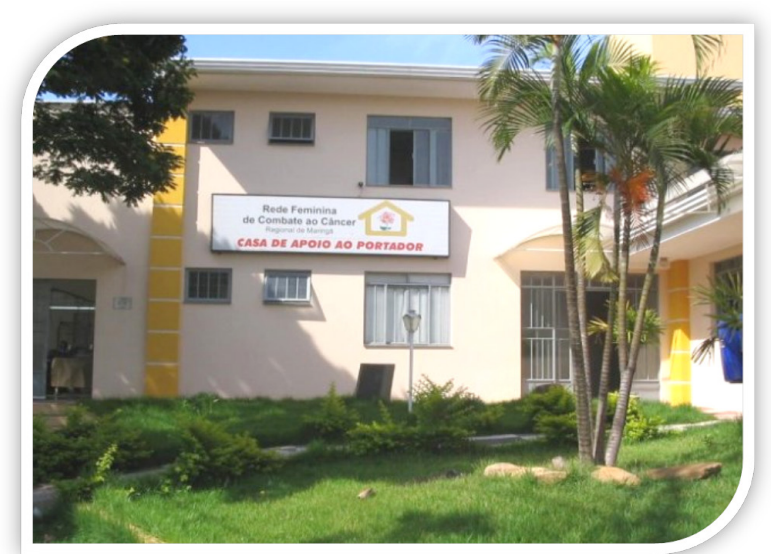

Figura 1. Sede da Rede Feminina de Combate ao Câncer no município de Maringá - PR

Diante do exposto, 0 objetivo da presente pesquisa foi identificar lesões e alterações bucais em pacientes pediátricos oncológicos cadastrados na Rede Feminina de Combate ao Câncer (RFCC) de Maringá/PR e aplicar métodos de atenção primária e curativa que se fizessem necessários, contribuindo para melhorar a qualidade de vida destes pacientes.

\section{MATERIAL E MÉTODOS}

0 estudo recebeu aprovação do Comitê de Ética em Pesquisa do UniCesumar por meio do protocolo $\mathrm{n}^{0}$ 1.474.425. Foi realizada uma amostra de conveniência em que foram incluídos no estudo uma totalidade de 22 crianças. Cada paciente recebeu uma visita, o que totalizou em 22 visitas domiciliares realizadas a pacientes oncopediátricos, cadastrados na Rede Feminina de Combate ao Câncer de Maringá - PR, com idades que variam entre dois e 14 anos. A RFCC é uma instituição filantrópica, sem fins lucrativos, que atende pessoas (crianças, jovens e adultos) em situação de vulnerabilidade social acometidas pelo câncer (Figura 1). Nas visitas foram incentivados os hábitos de higiene bucal e dieta saudável, e foi fornecida uma escova dental para cada criança de acordo com a sua idade (Figura 3). Aos pacientes que necessitavam de tratamento curativo, foi oferecido tratamento na Clínica de Odontologia do UniCesumar. Os dados obtidos foram analisados qualitativamente obtendo- 
se a frequência relativa das alterações encontradas, que foram: náusea, disfagia, mucosite, xerostomia, disgeusia, candidíase (Gráfico 1).

\section{RESULTADOS E DISCUSSÃO}

A discussão foi realizada com base nos dados coletados com 22 crianças e adolescentes em tratamento de câncer cadastrados na Rede Feminina de Combate ao Câncer do município de Maringá - PR.

Os dados empíricos coletados nas visitas domiciliares junto às crianças e aos adolescentes evidenciaram que $22(100 \%)$ apresentaram náuseas em decorrência do tratamento de câncer. Estes dados estão em concordância com 0 estudo realizado por Freitas e Neves (2013) e de Rolim et al. (2011) que relataram que o sintoma de náusea é comum em pacientes que realizam quimioterapia. Há semelhança com os dados relatados no estudo de Cruz e Giglio (2010) que salientam ser a náusea um dos sintomas presentes em pacientes que iniciam o tratamento do câncer.

Do total de pacientes avaliados neste estudo, $18(81,8 \%)$ relataram sintomas de disfagia. Santos et al. (2013) corroboram com este estudo evidenciando em sua pesquisa que um dos efeitos colaterais da radioterapia é a disfagia. Conforme Dragone (2010), a disfagia é definida como sendo a dificuldade de deglutir em pacientes que recebem irradiação na cabeça e no pescoço no tratamento de câncer, pois há falta de lubrificação do bolo alimentar. Rolim et al. (2011) explicam que a irradiação causa efeitos colaterais, pois como 0 tratamento ionizante não é seletivo (atua em células saudáveis) e se torna tóxico para 0 organismo, portanto, a irradiação provoca mudanças histofisiológicas na mucosa bucal, bem como provoca alterações estruturais e funcionais dos tecidos de suporte.

A mucosite oral foi diagnosticada por 16 $(72,7 \%)$ dos pacientes que fizeram parte deste estudo (Figura 2). A pesquisa realizada por Santos et al. (2011) demonstrou que pacientes submetidos à radioterapia ou à quimioterapia podem desenvolver reações na mucosa de diferentes intensidades. Conforme expõem Menezes et al. (2014), a mucosite oral é a condição resultante da inflamação da mucosa oral pela ação de medicamentos quimioterápicos ou radiação ionizante. Os autores salientam que aproximadamente 85 a 100\% dos pacientes submetidos a radio ou quimioterapia desenvolvem o quadro em graus variados, dependendo da dose de radiação recebida, do tipo de droga quimioterápica adotada e do regime de administração (SANTOS, 2011; MENEZES, 2014).

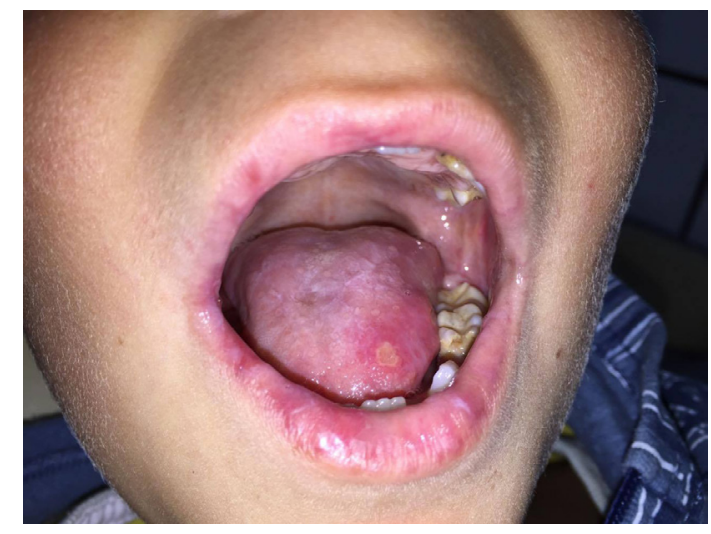

Figura 2. Mucosite oral

No presente estudo, a xerostomia foi relatada em 12 (54,5\%) crianças e adolescentes como sintoma do tratamento da quimioterapia e da radioterapia. Segundo Rolim et al. (2011), a xerostomia permanente é um dos efeitos tardios mais prevalentes em pacientes que foram submetidos ao tratamento de câncer. Na pesquisa realizada por Albuquerque et al. (2007) foi constatado que a xerostomia é uma manifestação bucal frequente após a radioterapia e quimioterapia, que se caracteriza pela redução qualitativa e quantitativa do fluxo salivar.

Das 22 crianças e adolescentes que foram avaliados neste estudo, dez relataram sintoma de disgeusia (45,4\%). A disgeusia é uma alteração onde ocorre a atrofia das papilas gustativas gerando a alteração da percepção dos sabores ácidos e amargo (ROLIM et al., 2011). Essas alterações da sensação são transitórias e reversíveis.

Do total de pacientes entrevistados, quatro $(18,1 \%)$ relataram ter desenvolvido candidíase oral 
como efeito colateral do tratamento de câncer. A candidíase trata-se de uma das infecções oportunistas mais frequentes nos pacientes em tratamento oncológico. Em indivíduos saudáveis, a Candida faz parte da microbiota oral residente (SENA, 2009), como microrganismo comensal, entretanto, em condições de imunossupressão ocorre um desequilíbrio, e esses micro-organismos passam a ser patogênicos (SENA, 2009). A Candida Albicans pode estar relacionada a quadros de baixa resistência do hospedeiro, doenças debilitantes e exposição à radiação (REDDING, 1999).

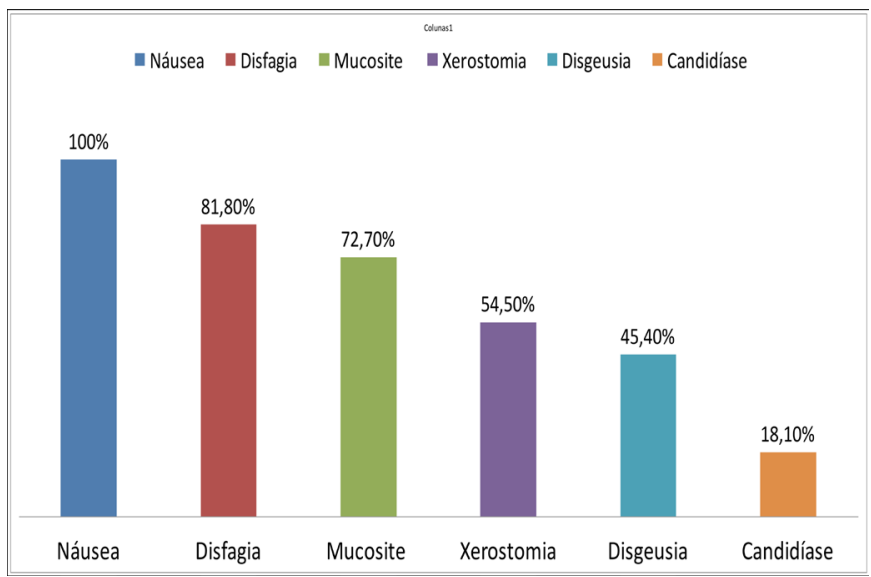

Quanto ao papel do cirurgião-dentista para a melhoria da qualidade de vida de pacientes oncológicos, a Academia Americana de Odontopediatria (AAPD) reconhece que o profissional da odontologia pediátrica desempenha um importante papel no diagnóstico, prevenção, estabilização e tratamento de problemas bucais e dentais que podem comprometer a qualidade de vida desses pacientes (AMERICAN ACADEMY OF PEDIATRIC DENTISTRY, 2014). A intervenção odontológica deve ser realizada com certas modificações, seguindo um protocolo que se baseia no histórico médico do paciente (AMERICAN ACADEMY OF PEDIATRIC DENTISTRY, 2014).

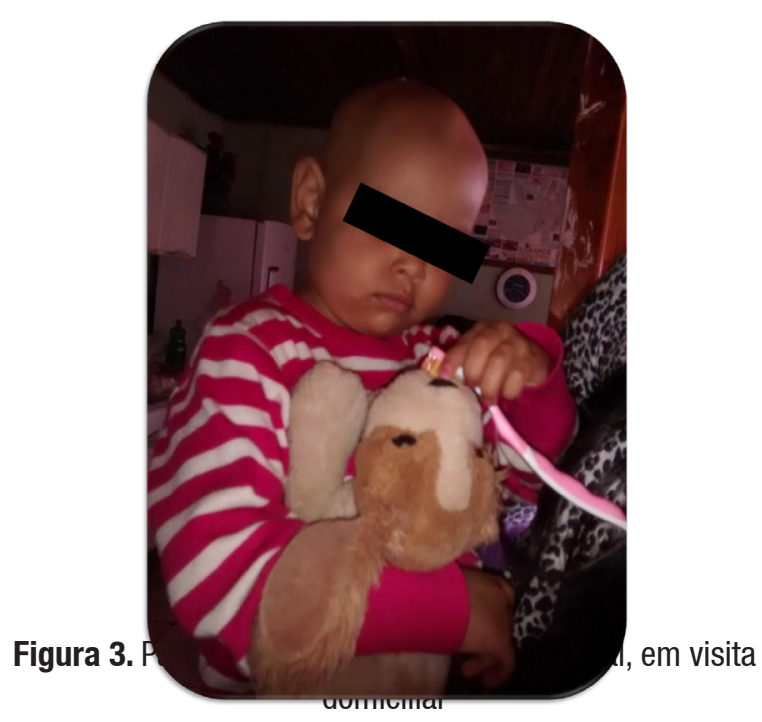

A cavidade oral é altamente susceptível aos efeitos de radiação e quimioterapia e é uma fonte frequente de infecçõesem pacientesimunossuprimidos. Frente a esta realidade, no início da intervenção dental, 0 dentista deve incluir medidas de higiene bucal, a fim de reduzir o risco de complicações orais e sistêmicas (SANTOS, 2003; CAIELLI, 1995).

\subsection{INSTRUÇÕES EM RELAÇÃO ÀS ALTERAÇÕES ENCONTRADAS}

Náusea: por se tratar de um sintoma multifatorial, o fracionamento da dieta; respeitando a vontade do paciente, bem como os gostos alimentares e os horários de alimentação; dando preferência para alimentos frios e com poucos condimentos; evitando frituras e alimentação rica em gordura e com odor muito forte. Orientar também quanto à higiene oral, principalmente após os episódios de vômito. Os pacientes devem ser instruídos a não permanecer em ambientes com odores que podem causar a sensação de náusea.

Disfagia: a dificuldade de deglutição é esclarecida pela carência de lubrificação do bolo alimentar, pela presença de infecções oportunistas e por dor na mucosa bucal (CRUZ, 2010). Diante disso, os pacientes devem estar cientes sobre a importância de manter a boca sempre umedecida por meio de ingesta hídrica, lubrificação labial com manteiga de cacau, 
utilização de substitutos de saliva e estimuladores de saliva (sialogogos) (VIEIR, 2005).

Mucosite oral: as ulcerações na mucosa bucal são muito comuns durante 0 tratamento antineoplásico (PAIVA, 2016). Os pacientes precisam ser instruídos a fazer a higienização com escova dental com cerdas ultramacias e higienizar com cautela (MENEZES, 2014). Realizar bochechos com água e bicarbonato até seis vezes ao dia (1/2 copo de água filtrada e 1 colher de café de bicarbonato de sódio). Orientar a ingesta de alimentos de consistência mais pastosa, em temperatura ambiente ou ligeiramente fria (WHITMYER, 1997). Evitar alimentos ácidos. Não fazer uso de soluções de enxágue bucal que contenham álcool. Para a prevenção da mucosite oral é preciso que, um dia após terminar a quimioterapia, sejam realizados bochechos com hidróxido de alumínio 10\%, cinco vezes ao dia, durante sete dias. A laserterapia também é utilizada na prevenção e no tratamento da mucosite oral (GONÇALVES, 2001).

Xerostomia: algumas medidas podem ser indicadas para promover alívio desse sintoma e minimizar suas consequências: uso tópico de flúor (para prevenção de cárie-dentária), saliva artificial, aumento da ingesta de água e uso de sialogogos (LIMA, 2003).

Ocasionalmente, algumas pessoas referem alívio sintomático com o uso de balas ou gomas de mascar sem adição de açúcar (LIMA, 2003).

Disgeusia: a xerostomia exacerba a alteração do paladar pelo espessamento do fluxo salivar. Recomenda-se, quando possível, fazer a escovação da língua com escova dental macia (isso ajuda a remover a camada espessa de saliva e, dessa forma, facilita 0 contato físico diretamente com 0 alimento (SANTOS, 2013).

Candidiase: recomenda-se Nistatina, suspensão oral, cinco vezes ao dia. É necessário fazer 0 bochecho com $5 \mathrm{~mL}$ da solução durante 15 dias, sendo indispensável a permanência do tratamento por mais uma semana após o fim dos sinais e sintomas (ALBUQUERQUE, 2007). Também é possível fazer uso de Miconazol a $2 \%$, gel oral. Aplicar na boca e/ou comissura labial quatro vezes ao dia (SANTOS, 2013).

A pesquisa teve uma amostragem de conveniência pequena, pois no município de Maringá não há tratamento público para 0 câncer infantil, com isso não se encontraram pacientes pediátricos que estivessem em tratamento, ou pós-tratamento. Observando-se que a grande maioria das crianças, quando diagnosticadas com câncer, era encaminhada para o município de Curitiba, para receber o tratamento. A resistência familiar em relação à importância dessa pesquisa foi um obstáculo, sendo que para um próximo estudo deve-se demonstrar melhor a importância do cirurgião-dentista ao tratamento oncológico, e como trazer benefícios ao paciente, para que seja aceito com mais frequência dentro das famílias a aplicação da pesquisa.

\section{CONCLUSÃO}

A partir da realização deste estudo pode-se concluir que

1) é fundamental a presença do cirurgião-dentista na equipe multidisciplinar oncológica;

2) 0 cirurgião-dentista deve intervir antes, durante e após o tratamento oncológico, minimizando os efeitos colaterais na cavidade oral;

3) apesar de 0 tratamento oncológico exigir ótimas condições bucais, nem todos os pacientes portadores de câncer têm acesso ao tratamento odontológico;

4) apesar da oferta de tratamento, percebeu-se resistência da parte de algumas famílias em relação ao papel do cirurgião-dentista para a saúde das crianças;

5) é fundamental que os responsáveis pelos pacientes sejam conscientizados sobre a importância da higiene bucal e da participação do cirurgiãodentista na equipe multiprofissional envolvida no tratamento contra o câncer;

6) a importância de identificar as lesões causadas pelo tratamento quimioterápico e radioterápico, e tratá-las quando necessário. 


\section{REFERÊNCIAS}

ALBUQUERQUE, R. A. de; MORAIS, V. L. L. de; SOBRAL, A. P. V. Protocolo de atendimento odontológico a pacientes oncológicos pediátricos - revisão da literatura. Revista de Odontologia da UNESP, v. 36 , n. 3, p. 275-280, 2007.

AMERICAN ACADEMY OF PEDIATRIC DENTISTRY. Clinical practice guidelines. Reference Manual, v. 37, n. 6, p. 15-16, 2014.

CACCELLI, É. M. N.; RAPOPORT, A. Para-efeitos das irradiações nas neoplasias de boca e orofaringe. Rev Bras Cir Cabeça Pescoço, v. 37, p. 198-201, 2008.

CAIELLI, C.; MARTHA, P. M.; DIB, L. L. Sequelas orais da radioterapia: atuação da odontologia na prevenção e tratamento. Rev Bras Cancerol., v. 41, p. 231-241, 1995.

CAMARGO, J. D. F.; BATISTELLA, F. I. D.; FERREIRA, S. L. M. Complicações Bucais Imediatas do Tratamento Oncológico Infantil: Identificação, Prevenção e Tratamento. JBP - Rev Iberoam0dontopediatrOdontol Bebê, v. 7, n. 36, p. 177184, 2004.

CORDOVA PEDERSEN, R.; CANCINO, C. M.; SASADA, I.; MARZOLA, C.; DILLENBURG, C.; HELLWIG, I. Protocolo de atendimento odontológico a pacientes oncológicos pediátricos: Relato de caso. Rev. Odontologia (ATO), Bauru, SP., v. 15, n. 12, p. 812-820, dez. 2015.

CRUZ, F. J. S. M.; GIGLIO, A. D. Prevenção de náusea e vômitos induzidos por quimioterapia. RBM Especial Oncologia, p.14-18, jul. 2010.

DRAGONE, M. L. S. Disfonia e disfagia: interface, atualização e prática clínica. Rev. soc. bras. fonoaudiol., São Paulo , v. 15, n. 4, p. 624-625, dec. 2010.

FINK, M. Vitamin D deficiency is a cofactor of chemotherapy-induced mucocutaneous toxicity and dysgeusia. Journal of Clinical Oncology, v. 29, n. 4, p. e81-e82, 2011.

FREITAS, B. N. de; NEVES, J. B. Efeitos colaterais da quimioterapia: os sentimentos apresentados pelos homens em tratamento. Revista Enfermagem Integrada - Ipatinga: Unileste, v. 6, n.1, jul./ago. 2013
GONÇALVES, R. C. C. Estudo de fatores de risco, prevenção e controle da mucosite oral radioinduzida. 2001. Tese (doutorado) - Fundação Antônio Prudente, São Paulo. 2001.

HALYARD, M. Taste and smell alterations in cancer patients-real problems with few solutions. Journal of Supportive Oncology, v. 7, n. 2, p. 68-69, 2009.

HOVAN, A.; WILLIAMS, M.; STEVENSON, P.; BRENNAN, M.; OHRN, K.; ELTING, L.; SPIJKERVET, F.; WAHLIN, Y. B. A systematic review of dysgeusia induced by cancer therapies. Supportive Care in Cancer, v. 18, n. 8, p. 1081-1087, 2010.

INSTITUTO NACIONAL DE CÂNCER (INCA). Estimativa 2012: incidência de câncer no Brasil (2011). Instituto Nacional de Câncer José Alencar Gomes da Silva, Coordenação Geral de Ações Estratégicas, Coordenação de Prevenção e Vigilância. Rio de Janeiro: Instituto Nacional do Câncer, 2011.

LIMA, A. A. S.; FIGUEIREDO, M. A. Z. de; LOUREIRO, M. S.; DUARTE, R. Radioterapia de neoplasias malignas na região decabeça e pescoço - 0 que 0 cirurgiãodentista precisa saber. Rev Odonto Ciência, v. 16, n. 33, p. 131-135, 2003.

MENEZES, A. C.; RAPOSO, B.; ALENCAR, M. J. S. Abordagem clínica e terapêutica da mucosite oral induzida por radioterapia e quimioterapia em pacientes com câncer. Rev. Bras. Odontol., Rio de Janeiro, v. 71, n. 1, jun. 2014.

PAIVA, M. D. E. B. et al. Complicações orais decorrentes da terapia antineoplásica. Arquivos em Odontologia, v. 46, n. 1, 2016.

PAIVA, M. D. E. B.; MORAES, J. J. C.;DE BIASE, R. C. C. G.; BATISTA, O. M.; HONORATO, M. C. T. M. Estudo retrospectivo das complicações orais decorrentes da terapia antineoplásica em pacientes do Hospital Napoleão Laureano-PB. Odontologia. Clin Científ., v. 6, n. 1, p. 51-55, 2007.

REDDING, S.; ZELLARS, R.; KIRKPATRICK, W.; MCATEE, R.; CACERES, M.; FOTHERGILL, A.; LOPEZ-RIBOT, J.; BAILEY, C.; RINALDI, M.; PATTERSON, T. Epidemiology of oropharyngeal Candida colonization and infection in patients receiving radiation for head and neck cancer. J Clin Microbiol, v. 37, p. 3896-3900, 1999. 
ROLIM, A. E. H.; COSTA, L. J. da; RAMALHO, L. Maria $P$. Repercussões da radioterapia na região orofacial e seu tratamento. Radiol Bras, São Paulo, v. 44, n. 6, p. 388-395, dec. 2011.

ROSA, L. N. da. Atenção estomatológica aos pacientes pediátricos oncológicos: submetidos a tratamento quimioterápico e à radioterapia. RGO, Porto Alegre, v. 45, n. 2, p. 111-114, mar./abr. 1997.

SALAZAR, M.; VICTORINO, F. R.; PARANHOS, L.Renato; RICCI, I. D.; GAETI, W. P.; CAÇADOR, N. P. Efeitos e tratamento da radioterapia de cabeça e pescoço de interesse ao cirurgião dentista. Revista 0donto., v. 16, n. 31, p. 62-68, 2008.

SANTOS, C. C. dos; NORO-FILHO, G. A.; CAPUTO, B. V.; SOUZA, R. C. de; ANDRADE, D. M. R. de; GIOVANI, E. M. Condutas práticas e efetivas recomendadas ao cirurgião dentista no tratamento pré, trans e pós do câncer bucal. Health Sci Inst., v. 31, n. 4, p. 368-372, 2013.

SANTOS, R. C. S.; DIAS, R. S.; GIORDANI,A. J.; SEGRETO, R. A.; SEGRETO, H. R. Comodo. Mucosite em pacientes portadores de câncer de cabeça e pescoço submetidos à radioquimioterapia. Rev. esc. enferm. USP, São Paulo, v. 45, n. 6, p. 1338-1344, dec. 2011.

SANTOS, V. I.; ANBINDER, A. L.; CAVALCANTE, A. S. Leucemia no paciente pediátrico: atuação odontológica. Cienc Odontol Bras., v. 6, p. 49-57, 2003.

SASSI, L. M.; MACHAD0, R. A. Protocolo préradioterapia de cabeça e pescoço. Rev Bras Cir Cabeça Pescoço, v. 38, n. 3, p. 2080-2090, 2009.

SENA, M. F.; GONDIM, L. A. M.; SOUZA, G. C. de Araújo; FERREIRA, M. Â. F.; LIMA, K. C. Tratamento de candidíase oral em pacientes com câncer de cabeça e pescoço: uma revisão sistemática. Revista da AMRIGS, Porto Alegre, v. 53, n. 3, p. 241-245, jul./set. 2009.

WHITMYER, C.C.; WASKOWSKI, J.C.; IFFLAND, H.A. Radiotherapy and oral sequelae: preventive and management protocols. J Dent Hyg, v. 71, p. 23-29, 1997.

WONG, H. M. Oral Complications and Management Strategies for Patients Undergoing Cancer Therapy.

The Scientific World Journal, v. 2014.
YURGEL, L. S. Manejo da Osteorradionecrose em Pacientes Submetidos à Radioterapia de Cabeça. Rev Odonto Ciência, v. 20, 47, 2005.

Recebido em: 07 de agosto de 2017 Aceito em: 26 de outubro de 2017 